\title{
A need for patient-centered care in managing patients with liver cirrhosis
}

\author{
Eileen L. Yoon \\ Department of Internal Medicine, Hanyang University Hospital, Hanyang University College of Medicine, Seoul, Korea
}

Keywords: Liver cirrhosis; Depression; Suicide; Patient-centered care

See Article on Page 283

Patients with new-onset liver cirrhosis frequently suffer psychologically from the stigma of an incurable disease, regardless of the presence of associated symptoms. Physically, patients initially present with acute decompensating events, such as jaundice, ascites, variceal hemorrhage, and overt hepatic encephalopathy. ${ }^{1}$ In addition, liver cirrhosis is notorious for having high rates of symptom prevalence beyond such decompensating events. The symptoms include pain (30-79\%), breathlessness (20-88\%), muscle cramps (56-68\%), insomnia (26-77\%), depression (4.5-64\%), and anxiety $(14-45 \%){ }^{2}$ These symptoms progressively deteriorate the quality of life of patients with cirrhosis. Frequent symptoms in patients with cirrhosis, such as fatigue, poor appetite, and sleep disturbance, can be overlooked by physicians, as they are related to physical discomfort rather than psychological problems. ${ }^{3}$ Treatment for liver cirrhosis has been limited to etiology-oriented and liver transplant-or-palliative care. ${ }^{4}$

In the current issue of Clinical and Molecular Hepatology, Jang et al. ${ }^{5}$ reported that patients with liver cirrhosis newly diagnosed had higher suicide rates. The suicide rate was 2.59 times higher in the first 2 years after diagnosis and 3.72 times higher in patients aged between 18 and 49 years compared to the rates in the matched cohort group. ${ }^{5}$ These suicide risks were similar between alcohol-related vs. non-alcohol-related, and compensated vs. decompensated groups. Previous studies on the association of liver cirrhosis and depression were based on a limited number of cases, ${ }^{6-9}$ self-reported liver disease, and suicidal ideation. ${ }^{10}$ They classified depression using either the Beck depression inventory or the Hamilton depression rating scale, ${ }^{8,9}$ which was far from the physician's diagnosis. ${ }^{3}$ The operational diagnosis of liver cirrhosis used in the current study may be clinically inaccurate. However, this study is unique in that it was based on a nationwide claims database. Moreover, the present study evaluated the association between liver cirrhosis and unambiguous objective cause of death, namely suicide. The suicide incidence rate in younger patients with liver cirrhosis was distinctively higher than that of the general population. According to the Korean Statistical Information Service, the number of self-harm cases that led to suicide was 26.9 per 100,000 persons, and it accounted for $4.7 \%$ of the total deaths in South Korea in 2019.1" The frequency of suicidal cases

\section{Abbreviations:}

$\mathrm{Cl}$, confidence interval; $\mathrm{OR}$, odds ratio

\section{Corresponding author : Eileen L. Yoon}

Department of Internal Medicine, Hanyang University Hospital, Hanyang University College of Medicine, 222-1 Wangsimni-ro, Seongdong-gu, Seoul 04763, Korea

Tel: +82-2-2290-8334, Fax: +82-504-159-7224

E-mail:mseileen80@gmail.com

https://orcid.org/0000-0003-0474-048X 
tends to increase in higher age groups; 67.8 per 100,000 persons in their 80 s, 46.2 per 100,000 persons in their 70 s, and 33 per 100,000 persons in their 60 s. ${ }^{12}$ The underlying mechanism for the association between the psychological substrate and liver cirrhosis remains unclear. Free triiodothyronine levels lower than 3.5 $\mathrm{mol} / \mathrm{L}$ among chronic hepatitis B patients have been associated with clinical depression (odds ratio [OR], 7.85; 95\% confidence interval $[\mathrm{Cl}], 1.839-33.547)$ and clinical insomnia (OR, 3.91; 95\% $\mathrm{Cl}, 1.417-10.789)$, respectively. ${ }^{13}$ Blood serotonin level was decreased in liver cirrhosis patients with depressive disorder. ${ }^{14,15}$ Disarrangement of melatonin homeostasis was presumed to be related to daytime sleepiness and fatigue. ${ }^{16}$ Nevertheless, further studies on the possible mechanisms are required.

These results support the need for patient-centered interventions to improve the quality of life for patients with liver cirrhosis. Paradoxically, the group with anxiety/depression showed longer overall survival than the non-disorder group among patients with hepatocellular carcinoma. ${ }^{17}$ Patients should be educated on how to take care of themselves during unpredictable decompensating events. They also need to be briefed on their treatment goals and how they can prepare for their future. ${ }^{18}$ Nonhospice palliative care for end-stage liver disease is frequently misconstrued as end-oflife care by both patients and healthcare providers. ${ }^{4}$ However, it is not far from clinical practice. Verna et al. ${ }^{4}$ suggested using screening tools to integrate palliative care intervention in the earlier stages of liver cirrhosis. Such tools included 1) Child-Pugh class C, 2) more than one liver-related admission within the last 6 months, 3) ongoing alcohol use in the context of known alcohol-associated liver disease, 4) unsuitability for liver transplantation, and 5) the World Health Organization/Eastern Cooperative Oncology Group performance score of 3 or $4 .{ }^{4}$ An ongoing multicenter randomized controlled trial aims to assess and compare the effectiveness of palliative care led by a specialist (non-hepatologist) and a trained hepatologist. ${ }^{18}$ The change in patients' quality of life in the first 3 months is the primary outcome. The results of this trial will provide scientific evidence supporting the need for integration of palliative care in routine hepatology care. Furthermore, evidencebased profit margins for palliative care services should be implemented in the healthcare system.

\section{Conflicts of Interest}

The author has no conflicts to disclose.

\section{REFERENCES}

1. D'Amico G, Morabito A, D'Amico M, Pasta L, Malizia G, Rebora $P$, et al. Clinical states of cirrhosis and competing risks. J Hepatol 2018:68:563-576.

2. Peng JK, Hepgul N, Higginson IJ, Gao W. Symptom prevalence and quality of life of patients with end-stage liver disease: a systematic review and meta-analysis. Palliat Med 2019;33:24-36.

3. Perng $C L$, Shen $C C$, Hu LY, Yeh CM, Chen MH, Tsai CF, et al. Risk of depressive disorder following non-alcoholic cirrhosis: a nationwide population-based study. PLoS One 2014;9:e88721.

4. Verma M, Tapper EB, Singal AG, Navarro V. Nonhospice palliative care within the treatment of end-stage liver disease. Hepatology 2020;71:2149-2159.

5. Jang SY, Rou WS, Kim SH, Lee BS, Eun HS. Association between new-onset liver cirrhosis and suicide risk in South Korea: a nationwide cohort study. Clin Mol Hepatol 2021;27:283-294.

6. Ko FY, Yang AC, Tsai SJ, Zhou Y, Xu LM. Physiologic and laboratory correlates of depression, anxiety, and poor sleep in liver cirrhosis. BMC Gastroenterol 2013;13:18.

7. Zhu HP, Gu YR, Zhang GL, Su YJ, Wang KE, Zheng YB, et al. Depression in patients with chronic hepatitis $B$ and cirrhosis is closely associated with the severity of liver cirrhosis. Exp Ther Med 2016;12:405-409.

8. Ko FY, Tsai SJ, Yang AC, Zhou Y, Xu LM. Association of CD8 T cells with depression and anxiety in patients with liver cirrhosis. Int J Psychiatry Med 2013;45:15-29.

9. Xu H, Zhou Y, Ko F, Ping J, Zhang J, Zhao C, et al. Female gender and gastrointestinal symptoms, not brain-derived neurotrophic factor, are associated with depression and anxiety in cirrhosis. Hepatol Res 2017:47:E64-E73.

10. Le Strat $Y$, Le Foll B, Dubertret C. Major depression and suicide attempts in patients with liver disease in the United States. Liver Int 2015;35:1910-1916.

11. Korean Statistical Information Service (KOSIS). Deaths according to the 50 etiologies in 2019. KOSIS web site, <https://kosis.kr/statH$\mathrm{tml} / \mathrm{statH}$ tml.do? orgld=101\&tblld=DT_1B34E01\&vw_cd=\&list_ $i d=\& s c r l d=\&$ seqNo $=\& l a n g \_m o d e=k o \& o b j \_v a r \_i d=\& i t m \_$ id=\&conn_path=K1>. Accessed 31 Dec 2020.

12. Korean Suicide Prevention Center. Current state of suicide according to the baseline characteristics in 2019. Korean Suicide Prevention Center web site, <https://spckorea-stat.or.kr/korea02.do>. Accessed 31 Dec 2020

13. Huang X, Zhang H, Qu C, Liu Y, Bian C, Xu Y. Depression and insomnia are closely associated with thyroid hormone levels in chronic hepatitis B. Med Sci Monit 2019;25:2672-2678.

14. Alekseeva AS, Beloborodova El, Rachkovskiy MI, Naumova EL, Lambrov EG, Philippova LP. Serotonin metabolism parameters in 


\section{CLINCAL and MOLECULAR}

patients with chronic hepatitis and liver cirrhosis. Bull Exp Biol Med 2008:146:577-579.

15. Chojnacki C, Walecka-Kapica E, Klupińska G, Wachowska-Kelly P, Żylińska K, Winczyk K, et al. Serotonin and melatonin secretion and metabolism in patients with liver cirrhosis. Pol Arch Med Wewn 2012;122:392-397.

16. Chojnacki C, Wachowska-Kelly P, Błasiak J, Reiter RJ, Chojnacki J. Melatonin secretion and metabolism in patients with hepatic encephalopathy. J Gastroenterol Hepatol 2013;28:342-347.
17. Lee KT, Lin JJ, Shi HY. Anxiety and depression are associated with long-term outcomes of hepatocellular carcinoma: a nationwide study of a cohort from Taiwan. World J Biol Psychiatry 2018;19:431439.

18. Verma M, Kosinski AS, Volk ML, Taddei T, Ramchandran K, Bakitas M, et al. Introducing palliative care within the treatment of end-stage liver disease: the study protocol of a cluster randomized controlled trial. J Palliat Med 2019;22:34-43. 